\title{
Irène Curie (1897-1956)
}

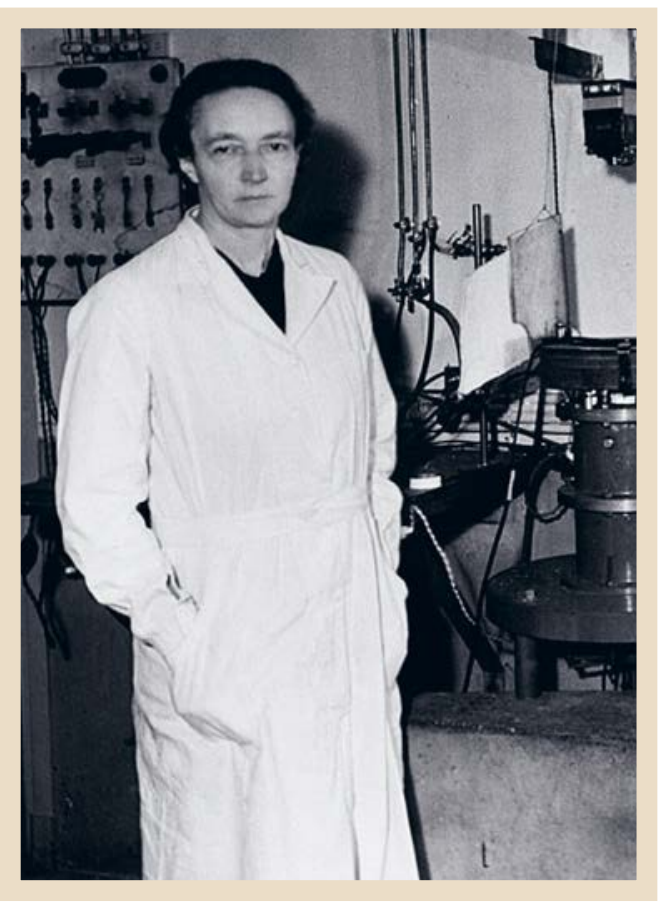

Par Claude Fabre

* claude.fabre@lkb.upmc.fr

Irène Curie nait à Paris en 1897. Elle est la fille ainée de Pierre Curie et Marie Sklodowska-Curie. Une année auparavant, Henri Becquerel a découvert un rayonnement étrange émis par les sels d'uranium. Marie décide d'en faire le sujet de ses travaux de thèse, bientôt suivie par Pierre, qui laisse de côté ses recherches sur le magnétisme.

\section{https://doi.org/10.1051/photon/202010123}

Article publié en accès libre sous les conditions définies par la licence Creative Commons Attribution License CC-BY (http://creativecommons.org/licenses/by/4.0), qui autorise sans restrictions l'utilisation, la diffusion, et la reproduction sur quelque support que ce soit, sous réserve de citation correcte de la publication originale.

I rène se trouve dès sa petite enfance dans un environnement d'exception, fait de parents très occupés pour cause de travaux de recherches chronophages, de fréquentation d'amis universitaires comme Jean Perrin, Paul Langevin, Aimé Cotton et Emile Borel, avec leurs enfants comme camarades de jeu, et de discussions scientifiques sur les derniers développements de la physique nucléaire. Irène évoluera sa vie durant dans ce milieu et ce groupe d'amis qui lui apportera beaucoup, tant sur le plan scientifique qu'affectif. Les Curie gardent du temps libre pour s'occuper de leurs enfants, Irène, et Eve née en 1904, et font le week-end de longues promenades en famille à bicyclette. Ils veillent avec bienveillance, affection et exigence à l'éducation de leurs filles. Irène a un an quand ses parents découvrent le polonium et le radium, 6 ans quand ils obtiennent le prix Nobel pour leurs travaux sur la radioactivité. Elle devient orpheline à 9 ans quand son père meurt à 47 ans dans un accident de la circulation. Elle a 14 ans quand sa mère obtient un deuxième prix Nobel, de Chimie celui-là pour ses travaux sur le polonium et le radium.

Pendant les années 1907 et 1908, son éducation se fait au sein de «La Coopérative », une sorte d'abbaye de Thélème fondée par Marie Curie et ses amis universitaires qui pratique un enseignement, hors $\bullet \bullet$

BIOGRAPHIE

\section{6}

Thèse sur la rayonnement alpha émis par le polonium

\section{3 \\ Découverte de la} «matérialisation » d'un photon gamma en une paire électron positron
1934

Découverte de la « radioactivité artificielle »

\section{5}

Prix Nobel

de Chimie avec

Frédéric Joliot
1945

Commissaire au Commissariat à l'Énergie Atomique 
École Républicaine, à la fois novateur et élitiste. Les cours y sont dispensés tour à tour par les parents, et quels parents, qu'on en juge : Marie Curie enseigne la physique, Jean Perrin la chimie, Paul Langevin les mathématiques, Henri Mouton, biologiste, les sciences naturelles, Edouard Chavannes, sinologue, l'histoire, J. Magrou, sculpteur, le dessin, etc. Beaucoup de temps est consacré à l'initiative et au travail personnel ainsi qu'aux pratiques sportives, artistiques et culturelles. Irène poursuit ensuite ses études secondaires au collège privé Sévigné dans le quartier latin. Ses parents ayant acheté une maison de villégiature à l'Arcouest près de Paimpol en Bretagne, elle y passe régulièrement ses vacances en famille, entourée de leurs amis et leurs enfants (la maison fut surnommée par les voisins « Sorbonne plage »), à s'adonner aux joies de la natation, de la voile, de la lecture et de l'étude. La correspondance qu'entretiennent Irène et sa mère montre toute la connivence, à la fois affective et intellectuelle, qui existe entre la mère et la fille. C'est donc tout naturellement qu'Irène suivra la voie ouverte par sa mère.

Lorsque la guerre éclate, Irène, à 17 ans, est bachelière. Elle com-

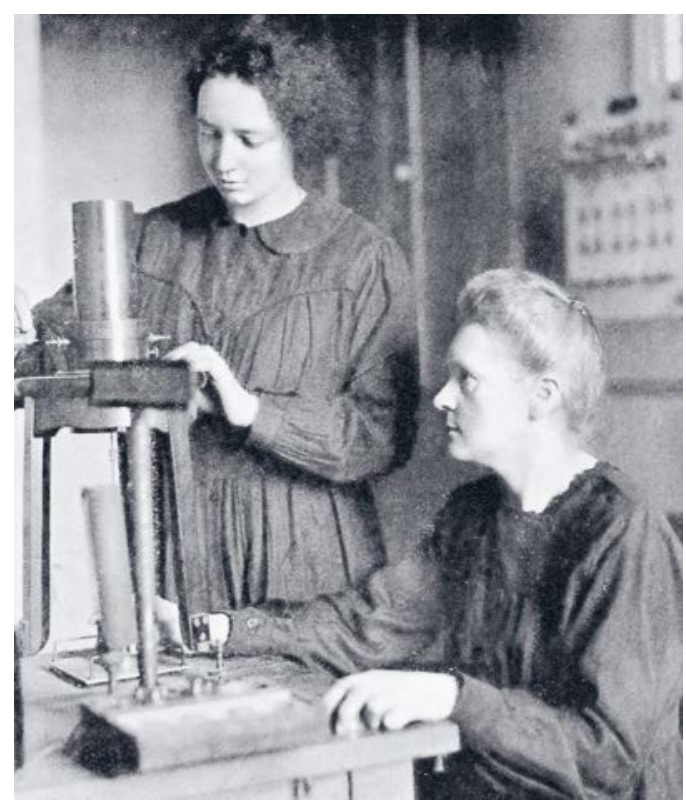

Irène et Marie Curie à l'Institut du radium (1925) (wikimedia) soutient sa thèse et épouse un jeune ingénieur collaborateur de sa mère, Frédéric Joliot, avec qui elle aura deux enfants, Hélène en 1927 et Pierre en 1932. Irène et Frédéric fusionneront bientôt leurs noms pour prendre celui de Joliot-Curie. Frédéric n'a pas de mal à s'insérer dans le cercle scientifique, familial et amical de «Sorbonne plage ». Parents et enfants se retrouvent chaque année à l'Arcouest pour des activités sportives, natation, randonnées..., entrecoupées de discussions et d'écriture d'articles scientifiques. La carrière d'une femme scientifique, même prix Nobel, est encore plus lente que celle des hommes : Irène est nommée en 1932 assistante, en 1937 maître de conférences puis professeur sans chaire, et n'obtiendra la chaire de physique générale et relativité (celle de sa mère, décédée en 1934) qu'en 1945 ! A la différence de son mari, elle ne sera jamais élue à l'Académie des Sciences.

À l'Institut du radium, ses recherches, pour la plupart effectuées en collaboration avec son mari Frédéric, s'inscrivent dans la continuité de celles effectuées par sa mère. Elles sont de caractère essentiellement expérimental, toujours à la pointe extrême mence ses études supérieures à la Faculté des Sciences de la Sorbonne. Sa mère met sur pied un service mobile de radiographie $\mathrm{X}$ : ce sont les $28 \mathrm{fa}$ meuses « petites Curie », des voitures transportant un appareillage de radiographie $\mathrm{X}$ au plus près $\mathrm{du}$ front et qui permet de repérer avec précision les éclats d'obus dans les chairs pour mieux guider les chirurgiens. Bien que très jeune, Irène s'engage à fond dans cette entreprise, accompagne souvent sa mère comme infirmière et contribue à l'entretien des appareillages. En 1917 elle forme elle-même les infirmières à cette nouvelle technique.

En 1918 elle reprend ses études supérieures à la Sorbonne et obtient en 1920 une licence de physique et une licence de mathématiques. Elle fréquente le nouvel Institut du radium dirigé par sa mère et lui sert d'assistante. Elle s'engage dans un travail de thèse sur les propriétés du rayonnement alpha émis par le polonium. 1926 est une année importante : elle des connaissances et dans un contexte international extrêmement compétitif. Elles ont recours à des techniques de caractérisation aussi bien physiques que chimiques. Les Joliot-Curie exploitent en particulier l'intense rayonnement de particules alpha émis par le polonium pour sonder la matière nucléaire. Au début des années 30, ils détectent dans leurs expériences un rayonnement qu'ils n'arrivent pas à expliquer, qui en fait trahit la présence d'un nouveau type de particule, le neutron. Les Joliot-Curie se font doubler sur le fil, puisque la mise en évidence définitive du neutron sera faite le mois suivant par Chadwick. A la suite de la découverte du positron, anti-particule de l'électron, la même année par Anderson dans les rayons cosmiques, le couple fait en 1933 une découverte importante en Photonique : il s'agit de l'observation de la création de matière à partir de lumière, qu'ils appelèrent " matérialisation ", dans laquelle un photon gamma d'énergie supérieure 
à $2 \mathrm{mc}^{2}$ rencontrant un noyau ( $\mathrm{m}$ étant la masse commune à l'électron et au positron) se transforme en une paire électron-positron. Ils observent aussi le phénomène inverse d'annihilation électron-positron avec production de rayonnement lumineux, qu'ils appelèrent « dématérialisation ».

En 1934, peu avant le décès de Marie, en bombardant des échantillons d'aluminium 27 avec des particules alpha, les Joliot Curie obtiennent du phosphore 30, élément radioactif inconnu dans la Nature. C'est le phénomène de radioactivité artificielle, pour laquelle ils obtiennent le prix Nobel de chimie 1935. La radioactivité artificielle permet la création d'une grande quantité de nouveaux radioéléments, qui seront très utiles par la suite en médecine, en chimie et en géologie. En 1938, en bombardant de l'uranium avec des neutrons, elle observe un nouvel élément, qui sera reconnu ultérieurement comme étant produit par fission, fission découverte à la fin de la même année par Meitner, Hahn et Strassmann.

Le petit monde de Sorbonne plage est de sensibilité « de gauche ». La montée du nazisme pousse Irène à s'engager en politique, notamment sous l'influence de Paul Langevin qui est très actif dans la défense de la paix. Elle adhère en 1934 au parti socialiste, en 1935 au comité de vigilance des intellectuels antifascistes et soutient les républicains espagnols face à l'agression franquiste. En 1936, c'est la victoire du Front Populaire : Irène est nommée sous-secrétaire d'état à la recherche scientifique dans le gouvernement Blum, poste qu'elle quitte au bout de quelques mois pour raisons de santé. C'est la première femme et le premier scientifique en France à occuper une fonction ministérielle. Son successeur à ce poste, Jean Perrin, autre prix Nobel, poursuivra son action et jettera les bases d'une organisation moderne de la recherche scientifique en France, avec la création du Centre National de la Recherche Scientifique, dont la mise en place sera interrompue par la guerre.

$\mathrm{Au}$ début de la guerre, Irène demeure à Paris aux côtés de Frédéric. Elle est atteinte de tuberculose et passe de nombreux mois en sanatorium dans les Alpes jusqu'à la Libération. Guérie, elle reprend alors son activité de recherche et de pilotage de la recherche: directrice de l'Institut du radium elle est nommée une des six Commissaires au Commissariat à l'Énergie Atomique nouvellement créé (Frédéric étant, lui, Haut-Commissaire). Elle participe en particulier au développement de la première pile atomique française Zoé. Elle s'investit dans le développement au sein de la nouvelle faculté d'Orsay d'un Centre de Physique Nucléaire disposant d'un synchrotron pour rester au meilleur niveau de la compétition internationale. Elle s'implique aussi dans la défense des droits des femmes.

Elle meurt à Paris en 1956 à l'âge de 58 ans d'une leucémie, la même maladie qui avait emporté sa mère, consécutive à une exposition accidentelle au polonium en 1946, ce même polonium qui était à l'origine des différents prix Nobel de la famille Curie. 